\title{
Processes leading to the coexistence of a host and its parasitoid in homogeneous environments: The role of an infected dormant stage
}

Marc Arancio $^{a, b, *}$, Marc Sourisseau $^{a}$, Sami Souissi $^{b}$

\author{
a IFREMER Brest, Laboratoire de Dynamique de L'Environnement Côtier. CS 10070, 29280 Plouzane. France \\ b UMR CNRS LOG 8187- Université Lille 1 Sciences et Technologies - Station Marine, 28 avenue Foch 62930 \\ Wimereux. France
}

*: Corresponding author : Marc Arancio, tel.: +33 298224465 ; email address : marc.arancio@ifremer.fr

\begin{abstract}
:
Theoretical studies have usually been used to explain host-parasitoid persistence in conditions of spatial heterogeneity or in homogeneous environments with specific conditions. In shallow estuaries where spatial heterogeneity is prevented by tides and river input, a common host-parasitoid system (dinoflagellate-Amoebophrya spp.) is able to persist even in the absence of specific conditions described in the literature. Recent observations have revealed that the cyst stage (during which the dinoflagellate host can survive in difficult environmental conditions in a dormant stage) can be infected by the parasitoid. The encystment/excystment process is suspected to be the basis for the long-term persistence of the system. In this work, the coexistence of Amoebophrya spp. and their hosts in homogeneous environments has been tested with an individual-based model of host-parasitoid interactions. Three processes that enable the coexistence were introduced into our model: (1) modifications in infection parameters, (2) a tritrophic food web and (3) a host encystment-excystment process. The persistence of the system was obtained in mixed conditions in all cases; however, the conditions required to obtain persistence with the infection parameter modifications were unrealistic. The tritrophic food web scenario produced short, stable, 10-d-long cycles in which the control of the parasite population in the environment was difficult to observe. The excystment process appears to be responsible for the interannual persistence of the system. Durable cycles with periods of $50 \mathrm{~d}$ were produced despite the unstable conditions. Moreover, these cycles did not depend on the proportion of infected cysts as long as a portion of the cysts remained healthy.
\end{abstract}

\section{Highlights}

Marine host-parasitoid study with Amoebophrya spp. and dinoflagellate hosts. The IBM model allows the introduction of a maturation time in host-parasitoid dynamics and gives good simulation of short in vitro dynamics. Short-term coexistence and oscillation in this ecosystem can be explained by a parasitoid population control. Interannual persistence in homogeneous environment of hostparasitoid system simulated with the excystment process.

Keywords: Individual-based model ; Dinoflagellate ; Parasitoid ; Amoebophrya ; Host-parasite interactions ; Sources of coexistence 


\section{Introduction}

Parasitism is one of the key types of interactions that can occur between two organisms. A specific category of parasite known as a parasitoid kills its hosts to survive and reproduce. Mechanisms allowing the host-parasitoid system to persist have been most studied in the insect community (Hassell et al. 1991, Kraaijeveld \& Godfray 1997, Stiling 1987). In these systems, experimental and theoretical studies generally predict unstable dynamics without incorporating external interactions or specific conditions in homogeneous environments into the relevant models (Getz and Mills (1996), May \& Hassel, 1981). Some heterogeneity is needed to enable these systems to persist, a feature that has been principally explained by patchy population distributions and different behvioral patterns of the host and parasitoid (Auger et al. 2000, Coats \& Bockstahler 1994, Hassell \& May 1988, Hassell et al. 1991, Hassell 2000, Murdoch \& Briggs 1996).

However, several of these persistent host-parasitoid systems have been found in mixed environments. Samples taken from mixed shallow estuaries have contained Syndiniales Amoebophrya spp., obligate parasitoids of dinoflagellates (Chambouvet et al. 2008, Coats et al. 1996). Amoebophrya species are widespread intracellular parasites that can infect more than 40 free-living dinoflagellate species (Park et al. 2004, Salomon et al. 2009). However, most isolated parasitoid strains are very specific and can infect only a few species or strains of host species. The infection begins when a dinospore (a biflagellate free-living form of Amoebophrya spp.) encounters and penetrates its host. Inside the host cell, the parasites develop into trophonts and use the cellular material of the host to grow. The trophont develops in the nucleus or in the cytosol of the host (depending on the species of host). Infection prevents host division and always leads to cell death. Maturation takes approximately two days and produces a multinucleate and multiflagellate stage known as a vermiform. After maturation, the vermiform ruptures through the host cell membrane and produces dozens to thousands of dinospores by cytokinesis after a short time (less than $1 \mathrm{~h}$ ) (Chambouvet et al. 2008, Coats \& Park 2002). A more in-depth description of the life cycle of Amoebophrya spp. was produced by Cachon (1964), Cachon and Cachon (1987) and Coats and Bockstahler (1994).

The conditions in which Amoebophrya and its host interact in mixed estuaries are, in theory, incompatible with the persistence of the system. Above all, the high infectivity and reproductive output of Amoebophrya should not allow the host population to maintain itself in 
the water column. The host can develop each year because it produces cysts that can survive for several years in the sediment. Cysts accumulate in the sediment and germinate in favourable conditions. The dinospores cannot survive without their hosts, yet hosts and parasitoids are observed every year. A more recent study (Chambouvet et al. 2011) suggested that parasitoids such as Amoebophrya spp. could infect their dinoflagellate hosts just before encystment (in the planozygote stage) and enter into dormancy within the host. The cyst stage represents a survival stage for both the host and the parasitoid that might be responsible for the long-term persistence of the system.

The aim of this paper was to study the effects of different processes that allow the coexistence of hosts and parasitoids (dinoflagellates and Amoebophrya, respectively) in a homogeneous environment. Three main processes were studied: the modification of infection parameters, the control of the parasite by grazers and the excystment process. An individual-based model (IBM) approach was developed to simulate short synchronous dynamics, thus overcoming the problem of rapid parasite generation time in a non-IBM model (Salomon \& Stolte 2010); in particular, a parasite maturation time was introduced into a phytoplankton ecophysiological model. The individual approach ensures that complex behviors and intracellular process modifications can be introduced into the model. The representation of the parasitoid-host dynamics (Amoebophrya spp. / dinoflagellate host) was primarily validated with experimental data from Coats and Park (2002) in an initial basic configuration. Three different hosts (Akashiwo sanguinea, Gymnodinium instriatum and Karlodinium veneficum) were used in the validation process. Subsequently, using the best simulated host-parasitoid couple, different processes that enabled coexistence were studied in three different model configurations: the basic configuration (validated with experimental data) with different parameterizations (model A), a configuration with a parasite grazer (model B; tritrophic model) and a configuration with hosts and parasites produced by cysts (model $\mathrm{C}$ ). The model description follows the ODD (Overview, Design concepts, Details) protocol for describing individual and agent-based models (Grimm et al. 2006, Grimm et al. 2010).

\section{Materials and methods}

\subsection{Purpose.}

The purpose of this model was to introduce a precise and unbiased maturation time with stochastic and changing biological processes (i.e., infection) into an ecophysiological model of the host and to simulate infection dynamics. 


\subsection{Entities, state variables, and scales.}

Host cells were individually simulated in a fixed volume of water and characterized by their cell age (d), size $(\mathrm{cm})$, carbon mass $\left(\right.$ pmolC.host $\left.^{-1}\right)$, infected state (true or false), number of infections, infection time (d) and type of death (natural or parasite-induced mortality). Depending on the model configuration, parasites concentrations ( $\mathrm{Par}$ - parasites. $\mathrm{ml}^{-1}$ ), grazers concentrations $\left(G\right.$ - grazers.ml-1) and non-individual host concentrations ( $H_{e}$ - hosts.ml-1) were also simulated. The time step used in the model is $30 \mathrm{sec}$. Simulations length ranged from $3.5 \mathrm{~d}$ to $150 \mathrm{~d}$.

\subsection{Process overview and scheduling.}

After initialization, at every time step (30 sec), each host cell undergoes an infection test and is subjected to growth and mortality processes. The parasite concentration is modified by parasite mortality and the number of infections. If a host cell becomes infected, its infected state changes, its growth stops and the parasite responsible for the infection is removed from the water concentration. At each time step until maturation, an infected cell can be re-infected (multi-infection) and subjected to the same mortality process as that of a healthy cell (i.e., density-dependent mortality with a carrying capacity). As soon as the maturation time is reached, the infected cell dies and produces $p_{\text {out }}$ parasites. The model is summarized in a schematic diagram (Fig. 1).

\subsection{Design concepts.}

The main parameters and state variables (carbon mass, maximum growth rate, maturation time) of host cells are randomly drawn from an empirical range of values (population parameter $\pm 12.5 \%$ ) of a uniform distribution at the initiation and during cell division to represent inter-individual variability. This assigned randomization avoids a numerical synchronization of cells (Hellweger \& Kianirad 2007, Kreft et al. 1998).

Cell infections are the main stochastic processes of the model and are determined at each time step for each host cell using the infection rate. Infections depend on the behavior of the host and parasite and on the infectivity and concentration of parasites. The infection process is further explained in the submodel section.

The outputs of the model were produced every 15 minutes. Host cell states, concentrations and death types and parasite concentrations were recorded.

\subsection{Initialization.}


Initial conditions were modified according to the model configuration and the simulation objective. For clarity reasons, the details about initialization are presented after the description of the different configurations in the section 2.8. Simulations.

\subsection{Input data.}

All of the simulations were carried out in nutrient replete conditions with non-limiting light at a temperature of $20^{\circ} \mathrm{C}$.

\subsection{Submodels.}

\subsubsection{Population variables.}

Because we assumed nutrient replete conditions, nutrient equations were not described. The changes in free dinospore concentrations (Par) were described by Equation (1):

$$
\operatorname{Par}(t+d t)=\underbrace{\operatorname{Par}(t) \exp \left(-m_{p} d t\right)}_{\text {natural motality }}+\underbrace{\frac{p_{\text {out }}}{V} \sum_{d t} N_{\text {mih }}}_{\begin{array}{c}
\text { productionby } \\
\text { infected cells }
\end{array}}-\underbrace{\frac{1}{V} \sum_{d t} N_{i}}_{\begin{array}{c}
\text { sucessful } \\
\text { infections }
\end{array}}
$$

where $m_{p}$ is the natural mortality rate of free parasites $\left(\mathrm{d}^{-1}\right), d t$ is the time step duration $(\mathrm{d}), V$ is the water volume $(2 \mathrm{ml}), p_{\text {out }}$ is the trophont size (number of dinospores produced by one infected host cell) inside each of the infected dead cells, $N_{\text {mih }}$ is the number of mature infected host cells that died from parasite lysis and $N_{i}$ is the number of dinospores that successfully infected host cells.

\subsubsection{Host growth.}

Host cell growth was estimated using a "deterministic cell size" $(m)$ as proposed by Hellweger and Kianirad (2007). Cell size was estimated by cell carbon (pmolC.cell ${ }^{-1}$ ) levels, and cell division occurred when $m \geq 2 m_{0}\left(m_{0}\right.$ is the minimal cell size of 75 pmolC.cell $\left.{ }^{-1}\right)$. For each cell, the following system is thus defined:

$$
\begin{aligned}
& \frac{d m}{d t}=\mu m \\
& \mu=l_{h} \mu_{\max }
\end{aligned}
$$

where $\mu_{\max }$ is the maximum growth rate $\left(\mathrm{d}^{-1}\right)$ and $l_{h}$ is the maximum limitation threshold.

\subsubsection{Host mortality.}


Two types of mortality were defined. mort $_{1}\left(\mathrm{~d}^{-1}\right)$ was estimated by a carrying capacity $\left(c_{\text {cell }}\right.$; hosts. $\mathrm{ml}^{-1}$ ) and represents all of the sources of mortality or population losses not caused by parasites. mort $_{1}$ is defined as follows:

$$
\text { mort }_{1}=\mu H / c_{\text {cell }}
$$

where $H$ is the host concentration. A carrying capacity of 10,000 hosts.ml ${ }^{-1}$ was chosen to reach a compromise between a realistic culture concentration and computing resource limitations. mort $_{2}\left(\mathrm{~d}^{-1}\right)$ was exclusively associated with parasitism and estimated by the number of infected cells that released parasites per day.

\subsubsection{Parasite model.}

The life cycle of Amoebophrya spp. was divided into two stages: the free-living (dinospore) stage in the water column and the vermiform (or trophont) stage in the host cells. The life span of dinospores (i.e., several days) is longer than that of the vermiform outside the host cell ( $<1$ h) (Coats \& Park 2002). An instantaneous cytokinesis was assumed to occur after host cell death, and trophonts were assumed to transform directly into dinospores. The maturation time $\left(t_{\text {mat }} ; \mathrm{d}\right)$, the number of produced parasites per host after maturation $\left(p_{\text {out }}\right.$; parasites.host $\left.\mathrm{t}^{-1}\right)$ and the dinospore mortality rate $\left(m_{p} ; \mathrm{d}^{-1}\right)$ were set according to the host and parasite species (see Table 1).

\subsubsection{Encounters and infections.}

In our model, for every successful infection, one free-living dinospore was removed from the parasite concentration (integrated during one time step; represented by $N_{i}$ in Eq. 1). Infections were conditioned by the infection rate $I$ (successful parasites.host ${ }^{-1}$.time step $^{-1}$ ) which is defined as follows:

$$
I=\beta \chi P a r
$$

where $\beta$ is the encounter kernel or host clearance rate $\left(\mathrm{ml} \cdot \mathrm{host}^{-1} \cdot \mathrm{d}^{-1}\right)$ and $\chi$ is the dinospore fixation probability (the probability of a dinospore infecting a host upon encounter; \%; see section 2.6.10).

Kiørboe and Saiz (1995) proposed a simple kernel based on the volume in which a predator and its prey can encounter each other. $\beta$ is used to estimate the probability of encounter of two particles and is associated with the swimming behavior and size of both organisms and the turbulence field. Havskum et al. (2005) applied this kernel to a model of a dinoflagellate 
prey-predator system (Fragilidium subglobosum/Ceratium tripos). We applied this kernel to our model of similarly sized microalgae. The kernel is defined as follows:

$$
\beta=\beta_{\text {turbulent }}+\beta_{\text {behaviour }}
$$

with:

$$
\beta_{\text {behaviour }}=\pi\left(r_{h}+r_{p}\right)^{2}\left(\frac{v_{p}^{2}+3 v_{h}^{2}}{3 v_{h}}\right)
$$

calculated with the radius and velocity of hosts $\left(r_{h}, v_{h}\right.$, respectively) and parasites $\left(r_{p}, v_{p}\right.$, respectively). During the experiments conducted by Coats \& Park, the culture media remained static and turbulence-free most of the time and was only briefly mixed before sampling to obtain representative samples. To simulate experimental conditions in the model, a still water hypothesis $\left(\beta_{\text {turbulent }}=0\right)$ and a homogeneous environment ( 0 dimension $)$ were set as parameters.

Hosts were assumed to swim in a straight path and parasites were assumed to be unable to swim. The final expression of behavior is:

$$
\beta_{\text {behaviour }}=\pi\left(r_{h}+r_{p}\right)^{2} \times v_{h}
$$

$r_{p}$ was fixed at $2.5 .10^{-4} \mathrm{~cm}$ and $v_{h}$ was fixed at $0.02 \mathrm{~cm} . \mathrm{s}^{-1}$ for the three species because of their fixed size and because of a lack of knowledge of instantaneous swimming speeds.

\subsubsection{Infection tests.}

Infection tests were carried out at each time step for every host cell with the relevant infection rate $(I)$. For values lower than $1, I$ can be assimilated to the host infection probability during that time step (i.e., infection probability.host ${ }^{-1}$.time step $^{-1}$ ). A short enough time step ensures infection rates of less than 1 . The maximum time step is defined as follows:

$$
d t_{\max }=\frac{1}{\beta \chi p_{\text {out }} c_{\text {cell }}}
$$

$p_{\text {out }} \times c_{\text {cell }}$ is the theoretical maximum parasitic concentration in the model. The minimum $d t_{\max }$ is $34.8 \mathrm{~s}$ for the species A. sanguinea; as a result, a time step of $30 \mathrm{~s}$ was chosen to fulfill the condition $I<1$ with the parameters and initial conditions used in this work. Subsequently infection was determined stochastically at each step with a random draw. If the uniform 
random draw $(\Omega=] 0 ; 1[$ ) was inferior to the infection probability (infection rate $I$ ), the tested cell became infected.

\subsubsection{Multi-infections.}

Multi-infections were observed in some experiments (Coats \& Park 2002) and are thought to have different effects on the development of the parasite; for example, such infections could increase or decrease the vermiform maturation time depending on the infected species (Coats $\&$ Park 2002). We considered that parasites could not develop when the cell was already infected because it was unclear what effects multi-infection had on infection and maturation time. Thus, multi-infection does not affect the host infection and only results in the death of other multi-infecting dinospores. Based on microscopic observations, a maximum of four multi-infections $\left(n i_{\max }\right.$; infection.host $\left.{ }^{-1}\right)$ could occur.

\subsubsection{Model B: Incorporation of a parasite grazer.}

The impact of grazing on Amoebophrya was tested with a tritrophic configuration. Predator pressure was added to the parasite population with Lotka-Volterra type equations. The chosen predator was a ciliate species (i.e., Strobilidium sp.) that was able to graze Amoebophrya spp. (Johansson \& Coats 2002). As a new state variable, the concentration of grazers $(G$; grazers. $\mathrm{ml}^{-1}$ ) was introduced in model A with the following equations:

$$
\begin{gathered}
\operatorname{Par}(t+d t)=\operatorname{Par}(t) \exp \left(-m_{p} d t\right)+\frac{p_{\text {out }}}{V} \sum_{d t} N_{\text {mih }}-\frac{1}{V} \sum_{d t} N_{i}-g d t G \text { Par } \\
G(t+d t)=G(t) \underbrace{\exp \left(\mu_{g}(\text { Par }) d t\right)}_{\text {growth }} \underbrace{\exp \left(-m_{g} d t\right)}_{\text {mortality }} \\
\text { with } \mu_{g}(\text { Par })=\mu_{\text {gmax }}\left(1-\exp \left(-k_{g} \text { Par }\right)\right)
\end{gathered}
$$

where $g$ is the grazer clearance rate $\left(0.864 \mathrm{ml}^{-1} \mathrm{~d}^{-1} \cdot\right.$ grazer $\left.^{-1}\right), m_{g}$ is the grazer mortality $\left(0.56 \mathrm{~d}^{-1}\right)$, $\mu_{g \max }$ is the maximum grazer growth rate $\left(1.8 \mathrm{~d}^{-1}\right)$ and $k_{g}$ is the parameter that defined the slope of the growth rate function $\left(6 \cdot 26.10^{-5} \mathrm{ml}\right.$.parasites $\left.{ }^{-1}\right)$. All of the parameters were provided by Johansson and Coats (2002) and Montagnes (1996).

\subsubsection{Model C: Excystment.}

In this configuration, excystment is added to model A. According to Chambouvet et al. 2011), some cysts are infected and produce parasites instead of healthy cells at cyst germination. Among the three species studied, only G. instriatum produced cysts; this species was thus 
used for all of the simulations of model C. Because of the chosen excystment rate, the small simulated volume (5 and $10 \mathrm{ml}$ ) and the IBM approach of the hosts, an intermediate state variable $\left(H_{e}\right)$ was created to simulate low host concentrations $\left(<1\right.$ cell. $\left.V^{-1}\right) . H_{e}$ is the concentration of hosts originating from excystment. Concentrations varied according to the following equation:

$$
\begin{gathered}
H_{e}(t+d t)=H_{e}(t) \exp (\mu d t)+\frac{h_{e} d t}{V}\left(1-c_{p}\right) \\
\operatorname{Par}(t+d t)=\operatorname{Par}(t) \exp \left(-m_{p} d t\right)+\frac{p_{\text {out }}}{V} \sum_{d t} N_{\text {mih }}-\frac{1}{V} \sum_{d t} N_{i}+\frac{1}{V} \underbrace{h_{e} d t c_{p} p_{\text {out }}}_{\begin{array}{c}
\text { parasite produced } \\
\text { byexcystement }
\end{array}}(
\end{gathered}
$$

where $h_{e}$ is the excystment rate (4000 to 8000 hosts. $\mathrm{m}^{-2} \cdot \mathrm{d}^{-1}$ ) and $c_{p}$ is the parasitic prevalence in the cysts (10 to $20 \%$ ).

When $H_{e}$ exceeds 1 cell. $V^{-1}$ (i.e., the minimum host concentration for individual simulation) one individual cell is created:

$$
\begin{aligned}
H_{e}(t) & =H_{e}(t)-1 / V \\
\text { and } H(t) & =H(t)+\underbrace{1 / V}_{1 \text { hostcell }}
\end{aligned}
$$

Excystment rates were found in the literature (Ishikawa and Taniguchi (1996), Ishikawa and Taniguchi (1997)) and the prevalence was set to values that corresponded to an advanced in situ infection of vegetative cells. The excystment rate was assumed to be constant because the influence of the parasite on encystment dynamics and the parasite's ability to "infect" cysts (i.e., planozygote infection just before encystment) are unknown.

\subsubsection{Experimental data and parameterization.}

Coats and Park (2002) provided an advanced analysis of the host-parasite dynamics of three host species (A. sanguinea, G. instriatum and $K$.veneficum) and their specific parasites of the same genus (Amoebophrya spp.) using data obtained from laboratory experiments. These experimental data were used to test the ability of our model to reproduce the maturation time and host-parasite dynamics for different host species. The parameterization was performed as independently as possible from the data used for the validation; however, some parameters had to be estimated from the infection dynamics (i.e., $p_{\text {out }}, \mu_{\max }$ and $t_{\text {mat }}$ ). The independent 
parameters were derived from one part of the experiments conducted by Coats and Park (2002) and from the literature (Nielsen (1996), Salomon and Stolte (2010)) at a temperature of $20^{\circ} \mathrm{C}$ and a salinity of 15 . Several parameters had to be calculated or modified because of the individual and stochastic nature of the model. The specific parameter sets are presented in Table 1.

The maturation time was modified to match the model specifications. Because of random modifications $( \pm 12.5 \%)$ in the model, the minimum time required for an infected host to produce parasites is $t_{m a} / 1.125$. Consequently, we estimated the maturation time differently from Coats and Park (2002) or Salomon and Stolte (2009). Using data from Coats and Park (2002), we estimated the minimum time required for the parasite density to surpass $10^{3}$ parasites. $\mathrm{ml}^{-1}$. The results are presented in Table 1 .

Salomon and Stolte (2010) estimated parasitoid reproductive outputs using data from the dynamics experiments conducted by Coats and Park (2002) without taking into account parasite mortality. Their outputs were thus greatly underestimated. In this study, an equation describing parasite concentrations taking parasite mortality into account was used to determine a new estimate of the parasite production $\left(p_{\text {out }}\right)$. At the beginning of parasite production (after the first wave of infection), this equation is as follows:

$$
\operatorname{Par}(t)=p_{\text {tot }}(\underbrace{(1-\exp (-k t))}_{\begin{array}{c}
\text { dinospores release } \\
\text { overtime }
\end{array}} \underbrace{\exp \left(-m_{p} t\right)}_{\text {parasitemortality }}
$$

where $p_{t o t}$ is the total parasite production for one generation of infected cells (number of dinospores) and $k$ is a host death rate $\left(\mathrm{d}^{-1}\right)$ representing the staggering of the parasite production. $p_{\text {out }}$ estimates were approximately 1,000, 1,000 and 80 parasites.host $^{-1}$ for A. sanguinea, G. instriatum and $K$. veneficum, respectively. The new production values were used in the simulation to validate the model and are presented along with parameters estimated in Eq. 17 in Table 1.

The dinospore fixation probability $(\chi)$ is a parameter that is very specific to the individual nature of the model and had to be calculated because it was never measured. The value of this probability was indirectly estimated from data produced by Coats and Park with a similar quantity, namely the dinospore population infection success $\left(s_{\text {exp }}\right)$. This dinospore population infection success, or the percentage of successful parasites among the total population, was used as follows to calculate $\chi$ with the final equivalence relationship (Eq. 20): 


$$
S_{\text {exp }}=\frac{\text { successful parasites }}{\text { total parasites }}=\frac{N_{h, t_{1}}}{N_{p, t_{0}}} \int_{t_{0}}^{t_{1}} I d t
$$

Where $t_{l}$ is the Coats and Park experiment end time (1.5 day), $N_{h, t_{1}}$ is the total host population at time $t_{l}, N_{p, t_{0}}$ is the total parasite population at $t_{0}$ and $\int_{t_{0}}^{t_{1}} I d t$ is the number of infections per host during the experimental period. Eq. 18 is combined with Eq. 5 to give:

$$
s_{\exp }=\frac{N_{h, t_{1}} \beta \chi}{N_{p, t_{0}}} \int_{t_{0}}^{t_{1}} \operatorname{Par}(t) d t
$$

Par is integrated during the infection and before the maturation of the infected cells; thus Par was defined without parasite production as follows:

$$
\operatorname{Par}(t)=\operatorname{Par}\left(t_{0}\right) \exp \left(-m_{p} t\right)-\frac{1}{V} \sum_{t_{0}}^{t} N_{i}(t)
$$

To resolve $\int_{t_{0}}^{t_{1}} \operatorname{Par}(t) d t$ we hypothesize a linear host population infection with $\frac{1}{V} \sum_{t_{0}}^{t} N_{i}(t)=\frac{s_{\text {exp }} \operatorname{Par}\left(t_{0}\right)}{t_{1}} t$ and $\operatorname{Par}\left(t_{0}\right)=N_{p, t_{0}} / V$. Eq. 18 is thus equivalent to:

$$
\begin{aligned}
& s_{\text {exp }}=\frac{N_{h, t_{1}} \beta \chi}{N_{p, t_{0}}} \times \frac{N_{p, t_{0}}}{V}\left(\left(1-\exp \left(-m_{p} t_{1}\right) / m_{p}\right)-\frac{1}{2} s_{\exp } t_{1}\right) \\
& \Leftrightarrow \chi=\frac{s_{\text {exp }}}{H\left(t_{1}\right) \beta} \times \frac{1}{\left(1-\exp \left(-m_{p} t_{1}\right) / m_{p}\right)-s_{\text {exp }} t_{1} / 2}
\end{aligned}
$$

$H\left(t_{l}\right)$ is the host concentration (hosts.ml $\mathrm{m}^{-1}$ ) at time $t_{1}$. Values of $s_{\exp }$ are presented in Table 1.

\subsection{Simulations.}

Five types of simulations were performed in this study: validation simulations, sensitivity tests and coexistence simulations with model A, model B and model C configurations.

Validation simulations were run with A. sanguinea, $G$. instriatum and $K$. veneficum parameterizations for $3.5 \mathrm{~d}$. Initial host and parasitoid concentrations were identical to the mean experimental densities (Table 2). 
A sensitivity test was conducted to identify the main parameters controlling the population dynamics. Oscillation tests were then carried out by modifying these parameters. The extinction time of the host population was modified to identify the parameters that maximize the life span of the host population and allow potential coexistence. Each simulation was run ten times for a duration of 20 days with initial host and parasite concentrations of 1,000 cells.ml ${ }^{-1}$. G. instriatum was used for all simulations.

In the model A coexistence tests, two parameters $\left(\chi\right.$ and $\left.p_{\text {out }}\right)$ were independently modified to assume values equal to $0.5 \%$ to $100 \%$ of their reference value (i.e., G. instriatum parameters). Simulations were run ten times for 150 days for each set of values. Initial host and parasite

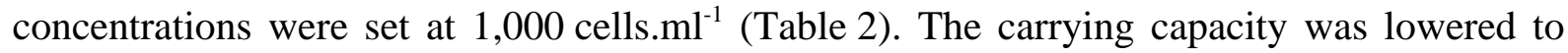
5,000 hosts. $\mathrm{ml}^{-1}$, a concentration closer to in situ values.

In the tritrophic configuration (model B), the system persistence was tested with three different grazer growth rates for 30 days. Three increasing values of $k_{g}$ were used to change and improve grazer growth. Values ranged from $6.26 \times 10^{-5}$ to $6.26 \times 10^{-4}$ ml.parasites ${ }^{-1}$. All simulations were performed with $G$. instriatum parameterization. The initial grazer concentration was arbitrarily fixed at 100 grazers. $\mathrm{ml}^{-1}$ because of the lack of relevant experimental data. Standard initial conditions did not provide enough resources to sustain the grazer concentration during the first maturation time. The initial prevalence of parasites was thus set at $10 \%$ to prevent infection synchronization and the large decline in the concentration of grazers at the beginning of the simulations. The carrying capacity was lowered to 5,000 hosts. $\mathrm{ml}^{-1}$.

In the excystment configuration (model C), different simulated volumes (5 and $10 \mathrm{ml}$ ) were tested to identify the simulations that were most consistent with the hypothesis of unlimited cysts in sediment. Initial concentrations were fixed at 0 . Host and parasite populations originated only from cysts. All simulations were performed with $G$. instriatum parameterization. Dynamics were simulated for 90 days.

\section{Results}

\subsection{Model response to experimental parameters and validation}

The model simulations and experimental data (Coats \& Park 2002) for each species are presented in Fig. 2, 3 and 4. In all cases, the principal dynamic events fit the experimental data. All of the simulations respect the parasite maturation time; no parasites were produced 
prematurely (Fig. 2c, 3c and 4c) and the different cohorts of parasites and infected hosts were clearly separated. The two waves of infection at a low parasite-host ratio (1:1) were correctly simulated (Fig. 2b, 3b and 4b). The host concentrations had similar orders of magnitude. The $\mathrm{R}^{2}$ for the infection concentration varied from 0.66 to 0.96 . The total infection of $A$. sanguinea and G. instriatum with a prevalence of $100 \%$ (Fig. 2e; 3e) and the strong but incomplete infection (96\% at 48 h) of K. veneficum (Fig. 4e) with high initial parasite concentrations were also represented in the simulations.

The differences between the model output and experimental results of all species are represented by the over- or under-estimation of the parasite concentrations (c and $\mathrm{f}$ graphics of Fig. 2, 3 and 4); these differences are associated with different production rates (slope) and a time lag (approximately $6 \mathrm{~h}$ ) in infection at the beginning of each infection. The time lag is particularly noticeable during the first ten hours (Fig. 2e; 3e) and at the beginning of the second wave of infection of $A$. sanguinea (1:1) (Fig. 2b; $60 \mathrm{~h}$ ). The model also underestimates the prevalence of parasites in A. sanguinea (1:1) at $24 \mathrm{~h}$ by $1 / 3$ (288 instead of 464 cells infected.ml ${ }^{-1}$; Fig. 2b). Healthy cells remain at a ratio of $20: 1$ after 24 h (Fig. 2d). An unexpected wave of mortality, that is most likely the result of infection, strikes the hosts during the second wave of infection at a low ratio and decreases the population by half (i.e., 790 instead of 1,560 hosts. $\mathrm{ml}^{-1}$ ). The parasite concentration in $K$. veneficum was underestimated at a low ratio and overestimated at a ratio of 40:1. Computed values are 20 times greater than experimental values at $40 \mathrm{~h}$ because of a lower predicted mortality $\left(22.55 \times 10^{3}\right.$ instead of $1.5 \times 10^{3}$ parasites. $\mathrm{ml}^{-1}$; Fig. 4 f). At a ratio of 40:1 (as was observed in the experiments), the model does not predict the infection of the entire population during the first wave of infection (96\% at $48 \mathrm{~h}$ ); however, the prevalence of the parasite is higher (87\% vs. $74 \%$ for experiments at $24 \mathrm{~h}$ ), and as soon as new parasites are produced, all of the remaining healthy hosts become infected (Fig. 4e). These differences suggest that the best parameterization is achieved with G. instriatum.

\subsection{Sensitivity test}

Results of the sensitivity test for the six tested parameters are shown in Fig. 5. The maximum growth rate $\left(\mu_{\max }\right)$, the parasite mortality $\left(m_{p}\right)$ and the maximum number of multi-infections $\left(n i_{\max }\right)$ did not affect (or only very slightly affected) the host extinction time. The trophont maturation time $\left(t_{\text {mat }}\right)$ has a positive and linear influence on the criterion (host extinction time). The parasite fixation probability $(\chi)$ and the dinospore production $\left(p_{\text {out }}\right)$ also have 
significant influence on the host extinction time but with negatively exponential relation. Only $\chi$ and $p_{\text {out }}$ were used in the coexistence tests because they naturally present more variability among the studied species.

\subsection{Coexistence in a basic system: Model A}

When the main two parameters $\chi$ and $p_{\text {out }}$ are modified, the model can simulate a total of five different dynamics (Fig. 6). Fig. 7 shows the transition among these five dynamics as a function of the variations in parameter. Parameters were assigned values ranging from $0.5 \%$ to $100 \%$ of their initial values. If the parameter value surpassed $100 \%$ of its reference value, the simulated outcome did not change. The most common scenario was the host extinction, which occurred when only one parameter was assigned values ranging from $3 \%$ to $100 \%$ of its reference value (Fig. 7). The other four dynamics appeared for lower values. The dynamics obtained were two types of unstable oscillations (i.e., the host extinction; Fig. 6a and the parasite extinction post-infection; Fig. 6b), stable oscillation (Fig. 6c), coexistence without oscillation (Fig. 6d) and parasite extinction (Fig. 6e). Values of $p_{\text {out }}$ lower than $1 \%$ of the original value or of $\chi$ lower than $0.7 \%$ of the original value were necessary to obtain persistence in the system.

\subsection{Coexistence with a grazer: Model B}

The introduction of a parasite grazer lowered the maximum parasite concentration and increased the maximum host concentrations in function of the grazer growth rate (modified through $k_{g}$; Fig. 8). The maximum parasite concentration decreased from $59 \times 10^{4}$ parasites. $\mathrm{ml}^{-1}$ without grazing to $3.32,0.97$ and $0.53 \times 10^{4}$ parasites. $\mathrm{ml}^{-1}$ with grazing and $k_{g}$ values of $6.26 \times 10^{-5}, 3.13 \times 10^{-4}$ and $6.26 \times 10^{-4} \mathrm{ml}$ parasites $^{-1}$, respectively. The maximum concentrations of hosts and grazers increased from 1,356 hosts. $\mathrm{ml}^{-1}$ to values ranging between 2,248 and 5,000 hosts. $\mathrm{ml}^{-1}$ and from 0 to values ranging from 399 to 719 grazers.ml ${ }^{-1}$. Even when $k_{g}$ was assigned low values (Fig. 8b), the parasite production levels and concentrations were high enough to completely infect the hosts (100\% parasite prevalence). With higher grazer growth rates, coexistence arose for periods of 10 to 12 days (Fig. 8c). At its highest value, $k_{g}$ yielded concentrations that converged and oscillated around stable mean values for a short period ( $\sim 52$ h; Fig. 8 d); this outcome is likely linked to the parasite maturation time.

\subsection{Coexistence with excystment: Model C}

The configuration with a production of hosts and parasites by excystment generates a nonconverging cyclic coexistence in all of the 500 simulations at $5 \mathrm{ml}$. The mean cycle duration 
is $44.8 \pm 5.0$ days in $5 \mathrm{ml}$ of water, with 8,000 hosts excysted. $\mathrm{m}^{-2}$.day ${ }^{-1}$ and a prevalence of $20 \%$. Strong heterogeneous blooms were simulated with maximum concentrations ranging from 46.8 to $8,772.8$ hosts. $\mathrm{ml}^{-1}$ and a mean concentration of 2,286 $\pm 1,919$ hosts. $\mathrm{ml}^{-1}$. This variability is associated with the stochasticity of the initial infection time ( $37 \pm 6.1$ days after the first host appearance) and explains the non-converging nature of this cycle. In the excystment model, all of the dynamics have similar values at the beginning of each development (i.e., 0 host. $\mathrm{ml}^{-1}$ and low parasite concentrations; point PE, Fig. 9). The initial infection time conditions the maximum concentration reached during the bloom: blooms with early initial infections will produce lower host concentrations, whereas blooms with late initial infections will reach high host concentrations.

An example infection cycle is presented in a phase diagram in Fig. 9. Dynamic events are strongly amplified and exaggerated because of the size of the simulated volume. The simulation begins with 0 hosts and 0 parasites. Initially, during the period of host growth, the parasite concentration reaches an equilibrium between production and mortality (0.32 parasites. $\mathrm{ml}^{-1}$; position PE in Fig. 9); during this period, the parasites are not able to infect cells. A decrease in the excystment rate or cyst prevalence modifies the parasite concentration equilibrium. As soon as an individual host is produced, the host concentration increases exponentially. The first infection ( $i_{1}$, Fig. 9) is visible with the loss of one parasite. $V^{-1}$ (at 3,065 hosts. $\mathrm{ml}^{-1}$ ) and a return to equilibrium during the maturation time. Three consecutive parasite generations follow the first infection until the death of all of the hosts nine days later. The initial infections of each parasite generation are indicated by $i_{2}, i_{3}$ and $i_{4}$. The host concentration reaches its maximum value (i.e., 6,640 hosts. $\mathrm{ml}^{-1}$ ) during the infection of the second generation of parasites. During the maturation time of each wave of infection, parasite production ceases and the concentration of parasites decreases. A decline in the host concentration without parasite production during the maturation of the third generation occurred because of unbalance between the growth rate $\left(\mu_{\max }\right)$ and the natural mortality $\left(\right.$ mort $\left._{1}\right)$ of the host. Most of the infected hosts are unable to reproduce; therefore, the net population growth rate decreases and the mortality of the host population (which is close to its carrying capacity) increases. After the third generation is produced and all of the hosts die, parasite concentrations return to equilibrium (PE) and a new cycle begins as soon as a host is produced by excystment.

A greater simulated volume decreases the initial infection times $(33.8 \pm 5.9$ days at $10 \mathrm{ml})$ and

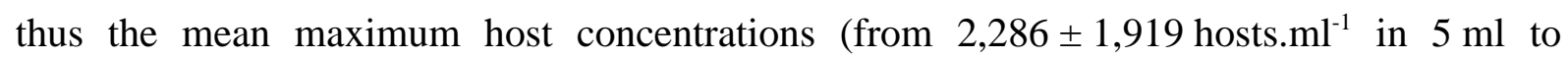


$1,371 \pm 1,121$ hosts. $\mathrm{ml}^{-1}$ in $10 \mathrm{ml}$ ) without interrupting the cyclic coexistence. The same result is obtained with an increased prevalence of the cyst population (increasing the infection probability); however, as long as some cysts remain uninfected, cyclic dynamics will occur.

\section{Discussion}

In this paper, the persistence of the host-parasitoid system on relatively short time-scale $(<2$ years) in a homogeneous estuarine ecosystem was investigated by modifying three different oscillatory factors. For this purpose, an IBM model was developed and parameterized for specific species. These species as well as some other dinoflagellates are well studied and known to be recurrently infected by the same parasite (Chambouvet et al. 2008, Coats et al. 1996, Velo-Suàrez et al. 2013). In the first step of this study, the model accurately simulated the different dynamics observed in laboratory experiments. Subsequently, the different model configurations produced oscillating dynamics in spite of the homogeneous environment.

\subsection{Simulation of in vitro dynamics, validation with experimental data}

The introduction of the parasite maturation time into an IBM ecophysiological model of the host was successful. The resultant model produced distinct waves of infection and no premature production of parasites. Moreover, the model was able to simulate the host and parasite extinction observed in laboratory experiments. Even with the use of a very simple encounter model with model parameterizations obtained from population measures, the dynamics were correctly simulated, with concentration values close to those observed in the experiments and a better adjustment than achieved in a non-IBM model (Salomon \& Stolte 2010).

The simple simulation of encounters was able to recreate all of the effects of density dependence observed in natural pathogen-host dynamics (Murdoch \& Briggs 1996). All of the simulated dynamics were influenced by this density dependence. First, modifications in cell density at a constant host:parasite ratio produced variations in the duration of the dynamics. Second, parasite mortality increased with multi-infections. Lastly, minimal host and/or parasite densities were needed to maintain the infections. All of the density-dependent effects were tested in the model (results not presented) and were found to be controlled by biological parameters (i.e., principally $\chi, \beta$ and $p_{\text {out }}$ ). Density dependence was realistically applied in all of the simulations and contributed to the good correspondence between model outputs and observed experimental dynamics. The small remaining biases presented in the results section 
were associated with inaccuracies in the encounter simulation and/or parameterization and with non-simulated biological processes.

The delay in infection observed at the beginning of each infection wave is the main biological process that was not represented in the model. In experiments, a time lapse is observed in parasite concentrations (at the end of the first infection) and in prevalence (during the second wave of infection). Coats and Park (2002) refer to this gap as an external maturation time during which parasites cannot infect hosts. For example, experimental parasite production in A. sanguinea (Fig. 2) began at $57 \mathrm{~h}$; in contrast, the experimental prevalence of the parasite increased only after $64 \mathrm{~h}$. For seven hours, newly formed parasites were not able to infect hosts and/or early infections could not be identified by the chosen staining techniques (Park et al. 2004). Because the high concentrations of parasites $\left(\sim 10^{6}\right.$ parasites. $\left.\mathrm{ml}^{-1}\right)$ rendered the simulation of individual parasites impossible, it was difficult to rigorously treat this time lag in the model.

The remaining differences between the model output and the experimental data arise from the parameterization. In particular the parameters that could not be estimated experimentally $(\chi$ and $\left.p_{\text {out }}\right)$. Both were calculated from population parameters $\left(s_{\text {exp }}\right.$ and $\left.m_{p}\right)$ and experimental data (see Eq. 10 and 12). This process thus compounded the uncertainty associated with all of the parameters used in the relevant equations and, because they are directly used in the encounter model, small estimation errors greatly affect the simulated dynamics. Other species-specific biases may be produced by inaccurate parameter estimates that are difficult to estimate experimentally. For example, the overestimation of the parasite concentration in K. veneficum (Fig 4f) could be explained by an underestimation of parasite mortality.

\subsection{Coexistence processes}

With appropriate parameterization, the model with the simplest configuration (model A) was able to simulate stable oscillations with periods of approximately 44 days. The parameter sets that produce these oscillations encompass a range of values in which the parasite growth rate is higher than the host growth rate (Getz and Mills (1996) but not high enough to cause unstable oscillations and host or parasite extinctions. These results show that even a parasite species with weak infectivity can persist in a system. However, the length of the oscillation periods is much longer than the observed in situ blooming window (Chambouvet et al. 2008, Mazzillo et al. 2011 and Wang et al. 2007); furthermore, the parameter values required to obtain stable oscillations are not realistic (Fig. 7) for the specific parasites used in this study 
(although they are encountered with certain strains of Amoebophrya that are able to infect suboptimal hosts). If the infection efficiency associated with a suboptimal host is low enough, the parasite could maintain a minimal concentration of infective units in the water column and coexist with this host with a low infection rate. The parasite could thus occupy the suboptimal host while waiting for its main host to develop.

The tritrophic configuration of the IBM model also enabled the coexistence of host and parasitoid species under realistic conditions and parameterizations. Only one type of tritrophic food web was tested in this study; however, other interactions such as host grazing (Rosenheim et al. 1995) or parasite/parasitoid competition (May \& Hassel 1981, Hogarth \& Diamond 1984) could lead to system persistence. The introduction of a parasite grazer also resulted in an extended intensity and duration of the host bloom (Fig. 8). These two results are consistent with observations made by other investigators (e.g., Johansson \& Coats 2002), with simulations of other marine models (Montagnes et al. 2008) and with theoretical tritrophic host-parasitoid-hyperparasitoid models (Beddington \& Hammond 1977 and May \& Hassell 1981). Short oscillations created by the addition of a grazer can thus be considered realistic. This phenomenon could explain the origin of oscillations ranging from 8 to 15 days that can be observed in a mixed shallow estuary such as the Penzé river. However, this type of oscillation can only be observed when the grazer growth is high enough to respond to changes in the parasite population.

The excystment process can also represent a source of oscillation, and even the strong infection of cyst populations can produce cycle dynamics from any initial conditions. The cycle is constituted by a host bloom of variable intensity followed by the bloom decay caused by infections and finally by a dormancy period of healthy and infected encysted hosts (not simulated). The encysted minimum residence time (several months according to Anderson; 1980) and the density dependence of the system are key factors that produce varying cycle dynamics as long as a portion of the cyst population remains healthy. The long residence time of the cysts ensures the removal of most of the parasite population by depriving them of their host. Then during the excystment, the hosts can develop because of the low encounter rate with parasites (proportional refuge; Hochberg \& Holt 1995). The host population can thus reach high concentrations before being infected. The encystment, triggered in situ by stressful conditions (e.g., high host concentrations, predation, nutrient limitation; Chambouvet et al. 2011, Toth et al. 2004, Wang et al. 2007) and the ability of the parasite to enter a dormancy period within its host (Chambouvet et al. 2011) allows parasites to survive during periods of 
low host abundance and cease propagation as soon as the cysts are formed. Encystment represents a refuge during conditions of poor host and parasite growth and causes inter-annual oscillatory dynamics. Some temporary cysts that may be resistant to the infection (Bravo \& Figueroa 2014) or not (Chambouvet et al. 2011) can also be produced. Although their residence time in the sediment is shorter than resting cyst (Bravo et al. 2010) it could be long enough to start a second infection cycle if the temporary cysts are resistant to parasites infections. However the mean bloom length (45 days) is too long and prevents successive development in the same year. The excystment process therefore cannot be the source of short in situ oscillations during the dinoflagellate bloom periods. Moreover, the minimum residence time of the resting cyst stage enables the inter-annual coexistence of the host-parasitoid system in an estuary, even when strong infection and host vegetative cell extinction occurs.

\section{Conclusion}

In this study, we investigated the influence of three parameters that allow host-parasitoid coexistence in homogeneous environments. Two sources (parameter modifications and tritrophic interactions) were previously studied in non-IBM models and similar results as those produced by our model were observed. Short-term coexistences were caused by agents or phenomena external to the host-parasitoid system that controlled the parasitoid population or weakly infective parasites. In this paper, the coexistence of these species of hosts was impossible with the range of parameter values estimated from experimental data without a parasite control. Because of the high decline in host concentrations during winter, classical processes (parameter modification and tritrophic interactions included) cannot explain the interannual persistence of the host-parasite system in homogeneous environments. However, the encystment-excystment process (during which the parasite does not divide or infect the host) allows the host and parasite to passively survive during the winter. To our knowledge, this process has not been investigated in previous theoretical studies. This process appears to effectively maintain the system with very infective parasites and represents a strategy commonly encountered in the marine dinoflagellate community. The sharing of a resistant form or the ability of a host-parasite couples to enter into dormancy at the same time (for example: dinoflagellate-Parvilucifera spp. system) should enable the persistence of several host-parasitoid systems and should be investigated in different hosts with a resting stage similar to the cyst stage of dinoflagellates. 


\section{Acknowledgments.}

We would like to thank Dr. Wayne Coats for providing and discussing the data set used in this study and Dr. Lourdes Velo-Suarez and Dr. Robin Raine, who helped with corrections. We also thank the anonymous reviewers for their constructive comments. This work was supported by PARALEX ANR project no. 2009-PEXT-01201.

\section{References}

Anderson, D. M., 1980. Effects Of Temperature Conditioning On Development And Germination Of Gonyaulax Tamarensis (dinophyceae) Hypnozygotes1. Journal of Phycology 16, 166-172.

Auger, P., Poggiale, J.-C., Charles, S., 2000. Emergence of individual behaviour at the population level. Effects of density-dependent migration on population dynamics. Comptes Rendus de l'Académie des Sciences - Series III - Sciences de la Vie 323, 119-127.

Beddington, J. R., Hammond, P. S., 1977. On the Dynamics of Host-Parasite-Hyperparasite Interactions. Journal of Animal Ecology 46, 811-821.

Bravo, I., Figueroa, R. I., 2014. Towards an Ecological Understanding of Dinoflagellate Cyst Functions. Microorganisms 2, 11-32.

Bravo, I., Figueroa, R. I., Garcés, E., Fraga, S., Massanet, A., 2010. The intricacies of dinoflagellate pellicle cysts: The example of Alexandrium minutum cysts from a bloomrecurrent area (Bay of Baiona, NW Spain). Deep Sea Research Part II: Topical Studies in Oceanography 57, 166-174.

Cachon, J., 1964. Contribution à l'étude des péridiniens parasites. Cytologie, cycles évolutifs. Ann Sci Nat Zool 6, 1-158.

Cachon, J., Cachon, M., 1987. Parasitic Dinoflagellates. In Taylor FJR (ed) The Biology of Dinoflagellates. Blackwell Sci. Publ, Oxford 21, 571-611.

Chambouvet, A., Alves-de-Souza, C., Cueff, V., Marie, D., Karpov, S., Guillou, L., 2011. Interplay Between the Parasite Amoebophrya sp. (Alveolata) and the Cyst Formation of the Red Tide Dinoflagellate Scrippsiella trochoidea. Protist 162, 637-649. 
Chambouvet, A., Morin, P., Marie, D., Guillou, L., 2008. Control of Toxic Marine Dinoflagellate Blooms by Serial Parasitic Killers. Science 322, 1254-1257.

Coats, D., Adam, E., Gallegos, C., Hedrick, S., 1996. Parasitism of photosynthetic dinoflagellates in a shallow subestuary of Chesapeake Bay, USA. Aquatic Microbial Ecology 11, 1-9.

Coats, D. W., Bockstahler, K. R., 1994. Occurrence of the Parasitic Dinoflagellate Amoebophrya ceratii in Chesapeake Bay Populations of Gymnodinium sanguineum. Journal of Eukaryotic Microbiology 41, 586-593.

Coats, D. W., Park, M. G., 2002. Parasitism Of Photosynthetic Dinoflagellates By Three Strains Of Amoebophrya (dinophyta): Parasite Survival, Infectivity, Generation Time, And Host Specificity. Journal of Phycology 38, 520-528.

Getz, W. M., Mills, N. J., 1996. Host-Parasitoid Coexistence and Egg-Limited Encounter Rates. The American Naturalist 148, 333-347.

Grimm, V., Berger, U., Bastiansen, F., Eliassen, S., Ginot, V., Giske, J., Goss-Custard, J., Grand, T., Heinz, S. K., Huse, G., Huth, A., Jepsen, J. U., Jørgensen, C., Mooij, W. M., Müller, B., Pe'er, G., Piou, C., Railsback, S. F., Robbins, A. M., Robbins, M. M., Rossmanith, E., Rüger, N., Strand, E., Souissi, S., Stillman, R. A., Vabø, R., Visser, U., DeAngelis, D. L., 2006. A standard protocol for describing individual-based and agentbased models. Ecological Modelling 198, 115-126.

Grimm, V., Berger, U., DeAngelis, D. L., Polhill, J. G., Giske, J., Railsback, S. F., 2010. The ODD protocol: A review and first update. Ecological Modelling 221, 2760-2768.

Hassell, M. P., 2000. Host-parasitoid population dynamics. Journal of Animal Ecology 69, 543-566.

Hassell, M. P., Comins, H. N., Mayt, R. M., 1991. Spatial structure and chaos in insect population dynamics. Nature 353, 255-258.

Hassell, M. P., May, R. M., 1988. Spatial heterogeneity and the dynamics of parasitoid-host systems. Ann. zool. fenn. 25, 55-61.

Hassell, M. P., May, R. M., Pacala, S. W., Chesson, P. L., 1991. The Persistence of HostParasitoid Associations in Patchy Environments. I. A General Criterion. The American Naturalist 138, 568-583. 
Havskum, H., Hansen, P. J., Berdalet, E., 2005. Effect of Turbulence on Sedimentation and Net Population Growth of the Dinoflagellate Ceratium tripos and Interactions with Its Predator, Fragilidium subglobosum. Limnology and Oceanography 50, 1543-1551.

Hellweger, F. L., Kianirad, E., 2007. Individual-based modeling of phytoplankton: Evaluating approaches for applying the cell quota model. Journal of Theoretical Biology 249, 554565.

Hochberg, M., Holt, R., 1995. Refuge evolution and the population dynamics of coupled hostparasitoid associations. Evolutionary Ecology 9, 633-661.

Hogarth, W. L., Diamond, P., 1984. Interspecific Competition in Larvae between Entomophagous Parasitoids. The American Naturalist 124, 552-560.

Ishikawa, A., Taniguchi, A., 1996. Contribution of benthic cysts to the population dynamics of Scrippsiella spp. (Dinophyceae) in Onagawa Bay, northeast Japan. Marine ecology progress series 140, 169-178.

Ishikawa, A., Taniguchi, A., 1997. In situ germination patterns of cysts, and bloom formation of some armored dinoflagellates in Onagawa Bay, north-east Japan. Journal of Plankton Research 19, 1783-1791.

Johansson, M., Coats, W. D., 2002. Ciliate grazing on the parasite Amoebophrya sp. decreases infection of the red-tide dinoflagellate Akashiwo sanguinea. Aquatic Microbial Ecology 28, 69-78.

Kiørboe, T., Saiz, E., 1995. Planktivorous feeding in calm and turbulent environments, with emphasis on copepods. Marine Ecology Progress Series 122, 135-145.

Kraaijeveld, A. R., Godfray, H. C. J., 1997. Trade-off between parasitoid resistance and larval competitive ability in Drosophila melanogaster. Nature 389, 278-280.

Kreft, J.-U., Booth, G., Wimpenny, J. W. T., 1998. BacSim, a simulator for individual-based modelling of bacterial colony growth. Microbiology 144, 3275-3287.

May, R. M., Hassell, M. P., 1981. The Dynamics of Multiparasitoid-Host Interactions. The American Naturalist 117, 234-261.

Mazzillo, F. F. M., Ryan, J. P., Silver, M. W., 2011. Parasitism as a biological control agent of dinoflagellate blooms in the California Current System. Harmful Algae 10, 763-773. 
Montagnes, D., 1996. Growth responses of planktonic ciliates in the genera Strobilidium and Strombidium. Marine Ecology Progress Series 130, 241-254.

Montagnes, D., Chambouvet, A., Guillou, L., Fenton, A., 2008. Responsibility of microzooplankton and parasite pressure for the demise of toxic dinoflagellate blooms. Aquatic Microbial Ecology 53, 211-225.

Murdoch, W. W., Briggs, C. J., 1996. Theory for Biological Control: Recent Developments. Ecology 77, 2001-2013.

Nielsen, M., 1996. Growth and chemical composition of the toxic dinoflagellate Gymnodinium galatheanum in relation to irradiance, temperature and salinity. Marine Ecology Progress Series 136, 205-211.

Park, M. G., Yih, W., Coats, D. W., 2004. Parasites and Phytoplankton, with Special Emphasis on Dinoflagellate Infections. Journal of Eukaryotic Microbiology 51, 145-155.

Rosenheim, J. A., Kaya, H. K., Ehler, L. E., Marois, J. J., Jaffee, B. A., 1995. Intraguild Predation Among Biological-Control Agents: Theory and Evidence. Biological Control 5, 303-335.

Salomon, P., Stolte, W., 2010. Predicting the population dynamics in Amoebophrya parasitoids and their dinoflagellate hosts using a mathematical model. Marine Ecology Progress Series 419, 1-10.

Salomon, S. P., Granéli, E., Neves, B. M. H. C., Rodriguez, G. E., 2009. Infection by Amoebophrya spp. parasitoids of dinoflagellates in a tropical marine coastal area. Aquatic Microbial Ecology 55, 143-153.

Stiling, P. D., 1987. The Frequency of Density Dependence in Insect Host-Parasitoid Systems. Ecology 68, 844-856.

Toth, G.B., Norén, F., Selander, E., Pavia, H., 2004. Marine dinoflagellates show induced life-history shifts to escape parasite infection in response to water- borne signals. Proceedings of the Royal Society of London. Series B: Biological Sciences 271 (1540), $733-738$. 
Velo-Suàrez, Lourdes, Brosnahan, Michael, L., Anderson, Donald, M., McGillicuddy, Jr., D. J., 2013. A Quantitative Assessment of the Role of the Parasite Amoebophrya in the Termination of Alexandrium fundyense Blooms within a Small Coastal Embayment. PLoS ONE 8, e81150.

Wang, Z.-H., Qi, Y.-Z., Yang, Y.-F., 2007. Cyst formation: an important mechanism for the termination of Scrippsiella trochoidea (Dinophyceae) bloom. Journal of Plankton Research 29, 209-218. 
Tables.

Table 1: Parameter sets used to validate the dynamics of different host-parasite couples and other calculations. Some of the parameters were derived from data produced by Coats and Park (2002), Salomon and Stolte (2010) and Nielsen (1996).

\begin{tabular}{|c|c|c|c|c|}
\hline Parameter & $\begin{array}{l}\text { Akashiwo } \\
\text { sanguinea }\end{array}$ & $\begin{array}{l}\text { Gymnodinium } \\
\text { instriatum }\end{array}$ & $\begin{array}{c}\text { Karlodinium } \\
\text { veneficum }\end{array}$ & Unit \\
\hline$p_{\text {out }}$ & 1085 & 1026 & 84 & parasites.host ${ }^{-1}$ \\
\hline$m_{p}$ & 0.48 & 1.02 & 0.26 & $\mathrm{~d}^{-1}$ \\
\hline$\mu_{\max }$ & 0.21 & 0.24 & 0.63 & $\mathrm{~d}^{-1}$ \\
\hline$l_{h}$ & 0.9 & 0.9 & 0.9 & $\%$ \\
\hline$c_{\text {cell }}$ & 10 & 10 & 10 & $10^{3}$ hosts. $\mathrm{ml}^{-1}$ \\
\hline$t_{m a t}$ & 59 & 54 & 50 & $\mathrm{~h}$ \\
\hline$\beta$ & 0.0275 & 0.0573 & 0.0054 & $\mathrm{ml} \cdot \mathrm{d}^{-1} \cdot \operatorname{host}^{-1}$ \\
\hline$\chi$ & 0.83 & 0.28 & 1.41 & $\%$ \\
\hline$r_{h}$ & 20.0 & 30.0 & 7.5 & $\mu \mathrm{m}$ \\
\hline$r_{p}$ & 2.5 & 2.5 & 2.5 & $\mu \mathrm{m}$ \\
\hline$v_{h}$ & 200 & 200 & 200 & $\mu \mathrm{m} \cdot \mathrm{s}^{-1}$ \\
\hline$S_{\text {exp }}$ & 18 & 9 & 9 & $\%$ \\
\hline$n i_{\max }$ & 4 & 4 & 4 & parasites.host ${ }^{-1}$ \\
\hline$p_{t o t}$ & 413 & 739 & 14 & $10^{3}$ parasites. $\mathrm{ml}^{-1}$ \\
\hline$k$ & 5.53 & 10.70 & 4.61 & $\mathrm{~d}^{-1}$ \\
\hline
\end{tabular}

Table 2: Initial simulation conditions. Numbers below the species name represent the parasite-host ratio reported by Coats and Park (2002).

validation

coexistence tests

\begin{tabular}{|c|c|c|c|c|c|c|c|c|c|}
\hline \multirow{2}{*}{ species / model: } & \multicolumn{2}{|c|}{ A. sanguinea } & \multicolumn{2}{|c|}{ G. instriatum } & \multicolumn{2}{|c|}{ K. veneficum } & \multirow{2}{*}{ Model A } & \multirow{2}{*}{ Model B } & \multirow{2}{*}{ Model C } \\
\hline & $1: 1$ & $20: 1$ & $1: 1$ & $115: 1$ & $1: 1$ & $40: 1$ & & & \\
\hline $\begin{array}{l}\text { Host initial density } \\
\left(\text { cells.ml }{ }^{-1}\right)\end{array}$ & 1094 & 1125 & 746 & 720 & 947 & 1012 & 1000 & 1000 & 0 \\
\hline $\begin{array}{l}\text { Parasites initial } \\
\text { density }\left(10^{3} \text { cells } \cdot \mathrm{ml}^{-1}\right)\end{array}$ & 1.625 & 18.75 & 0.875 & 71 & 1 & 40.5 & 1 & $\begin{array}{c}\text { prevalence } \\
10 \%\end{array}$ & 0 \\
\hline
\end{tabular}


Figure captions.

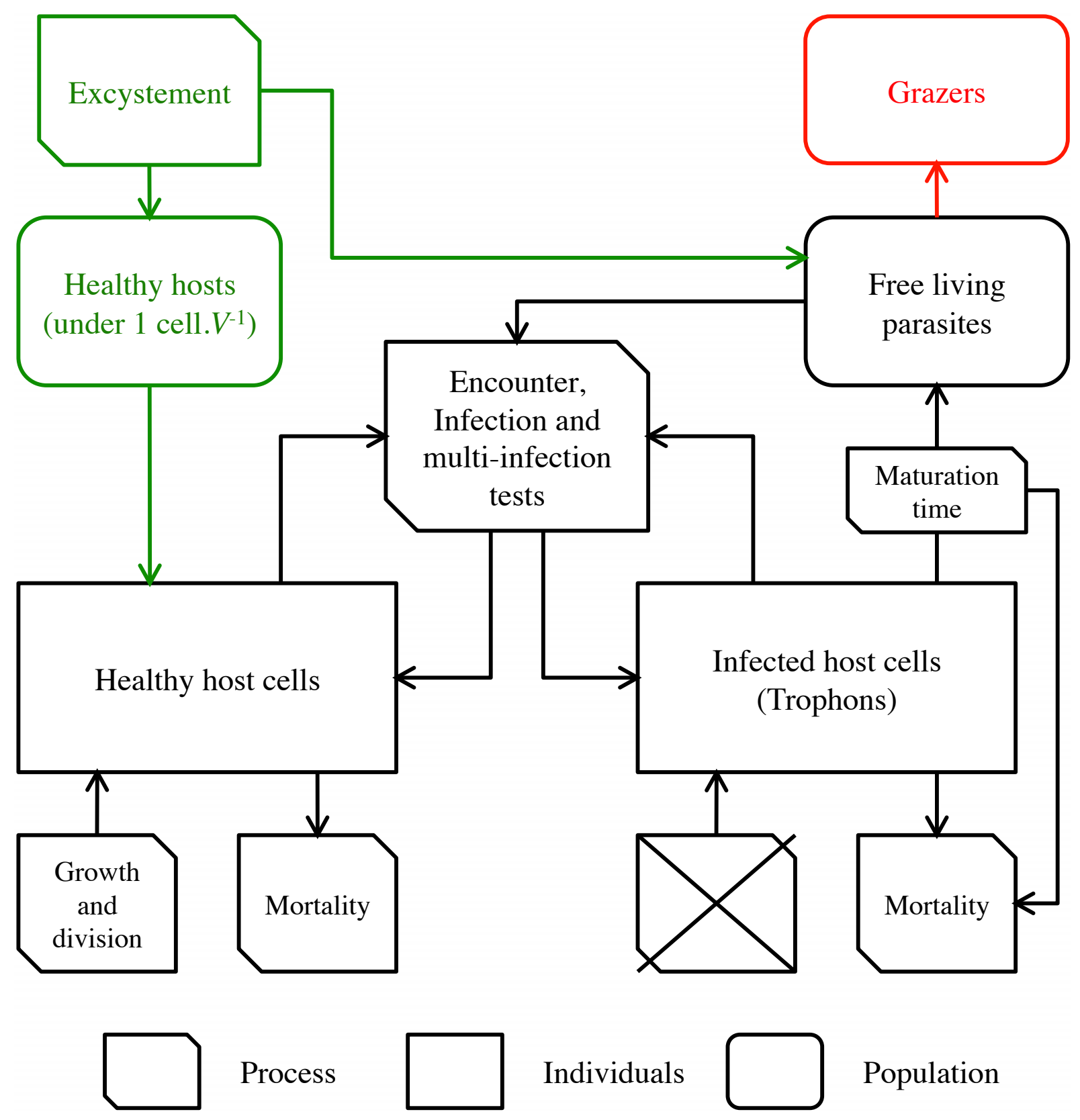

Fig. 1: Schematic diagram of the IBM model structure at each time step. Each part of the model that is interacting with another part of the model during a time step is represented by its category. Squares represent individually simulated host cell populations (either healthy or infected). Squares with rounded corners represent population variables that were not individually simulated. Squares with cut corners represent all biological and physical processes linking to and interacting with individual and population-level variables. Black compartments represent model A, black and red compartments represent model B and black and green compartments represent model C. 

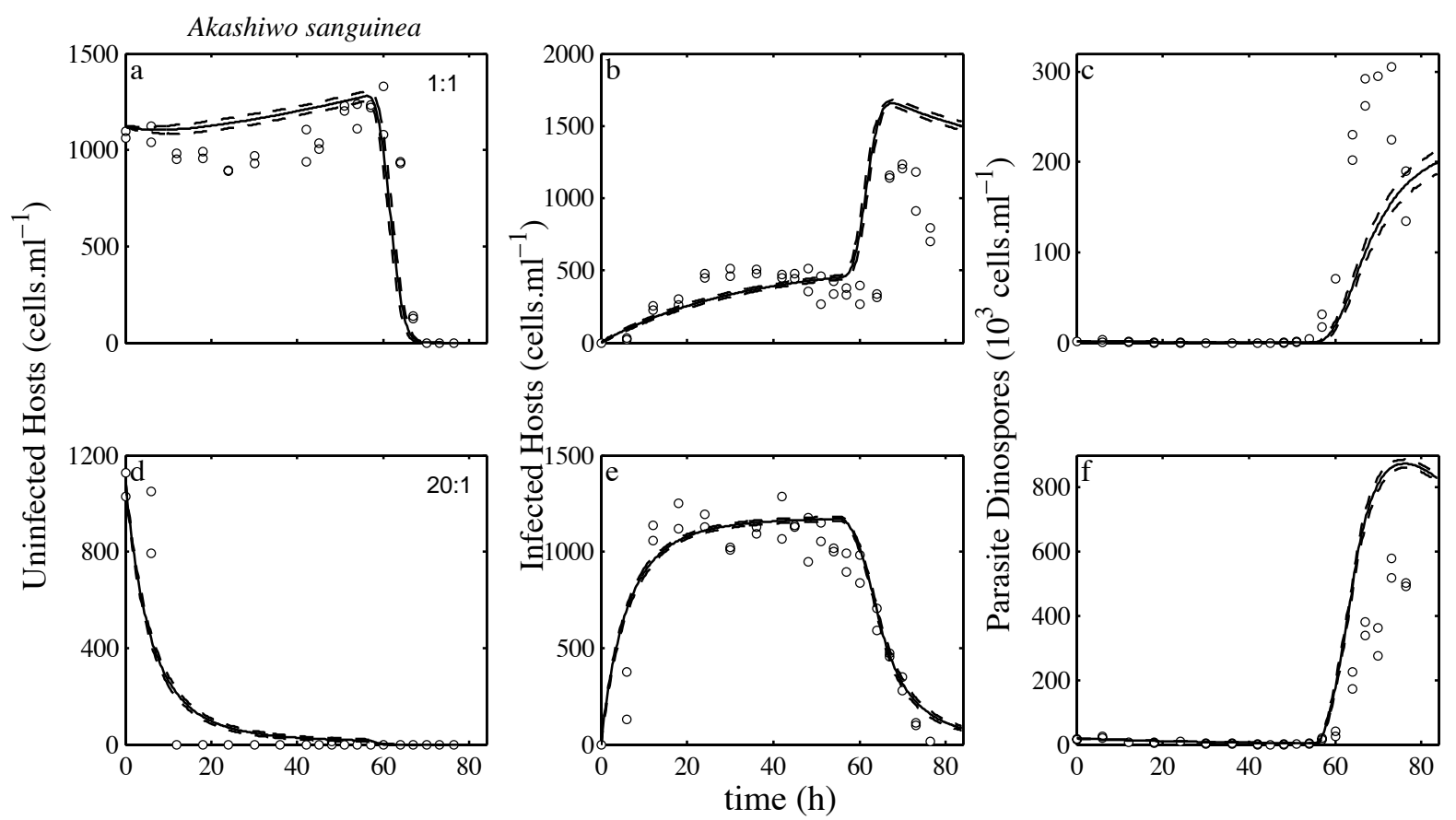

Fig. 2: Time series of the infection of Akashiwo sanguinea by Amoebophrya spp. Experimental data (open circles) from Coats \& Park (2002) and model simulations (black lines) with a 99 percentile confidence interval (dashed lines). Healthy host concentrations (a and $d$ ), infected host concentrations ( $b$ and e) and parasite concentrations (c and f). Differences in parasite-host ratios are indicated in the top right corner of a and d. 

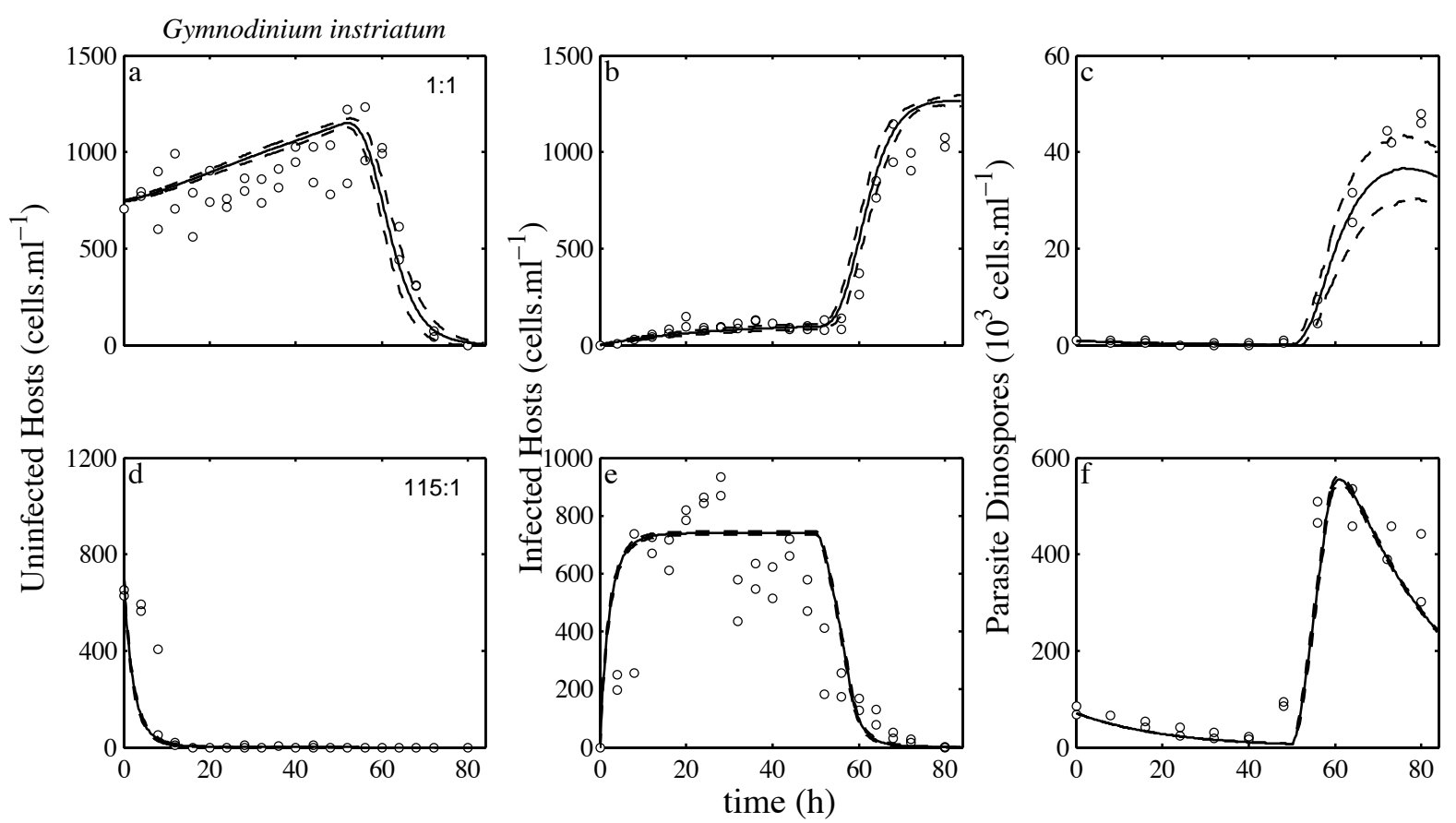

Fig. 3: Time series of the infection of Gymnodinium instriatum by Amoebophrya spp. Experimental data (open circles) from Coats \& Park (2002), model simulations (black lines) with a 99 percentile confidence interval (dashed lines). Healthy host concentrations (a and d), infected host concentrations (b and e) and parasite concentrations (c and f). Differences in parasite-host ratios are indicated in the top right corner of a and $\mathrm{d}$. 

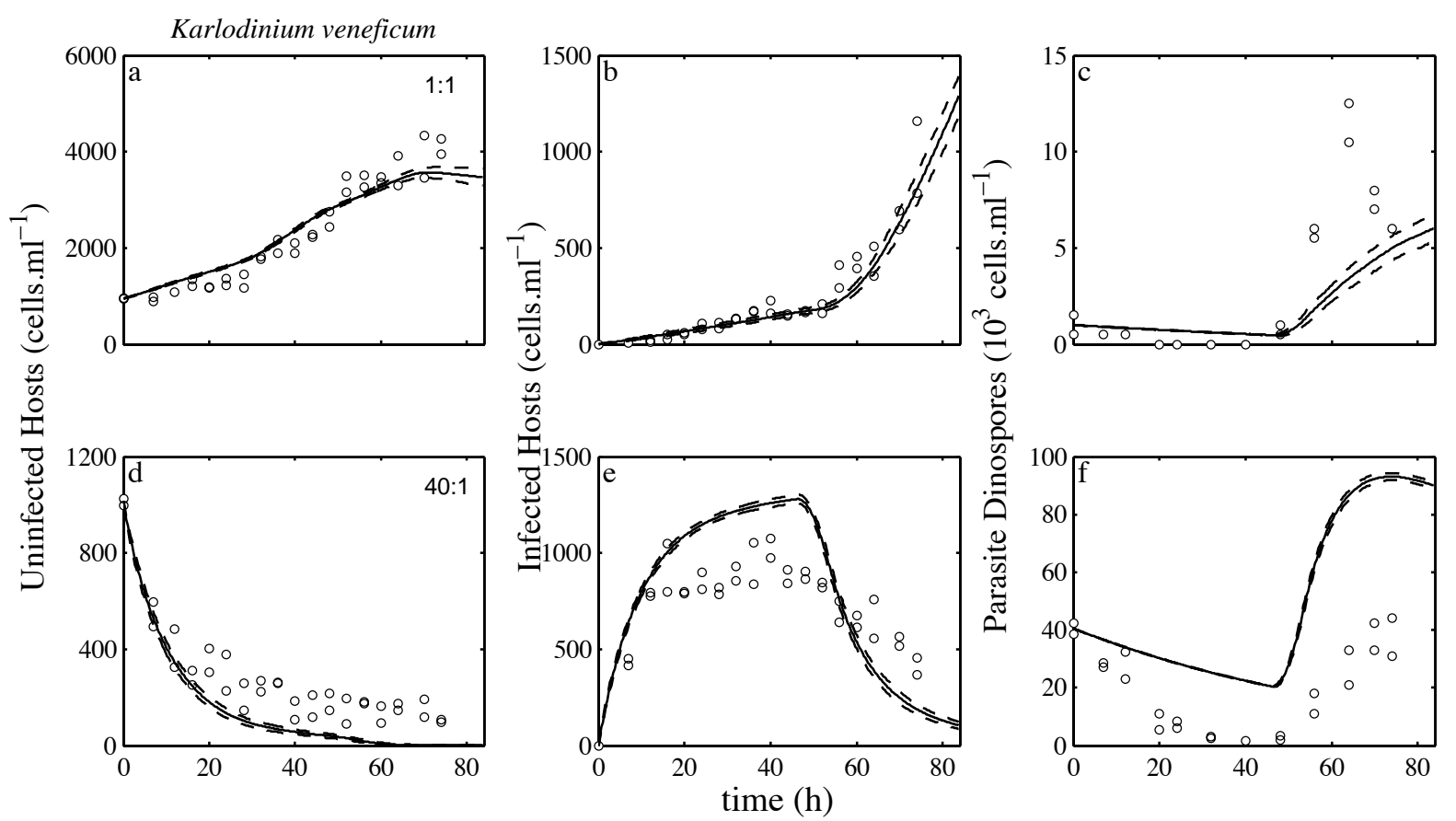

Fig. 4: Time series of the infection of Karlodinium veneficum by Amoebophrya spp. Experimental data (open circles) from Coats \& Park (2002) and model simulations (black lines) with a 99 percentile confidence interval (dashed lines). Healthy host concentrations (a and $d$ ), infected host concentrations ( $b$ and e) and parasite concentrations (c and f). Differences in parasite-host ratios are indicated in the top right corner of a and d. 


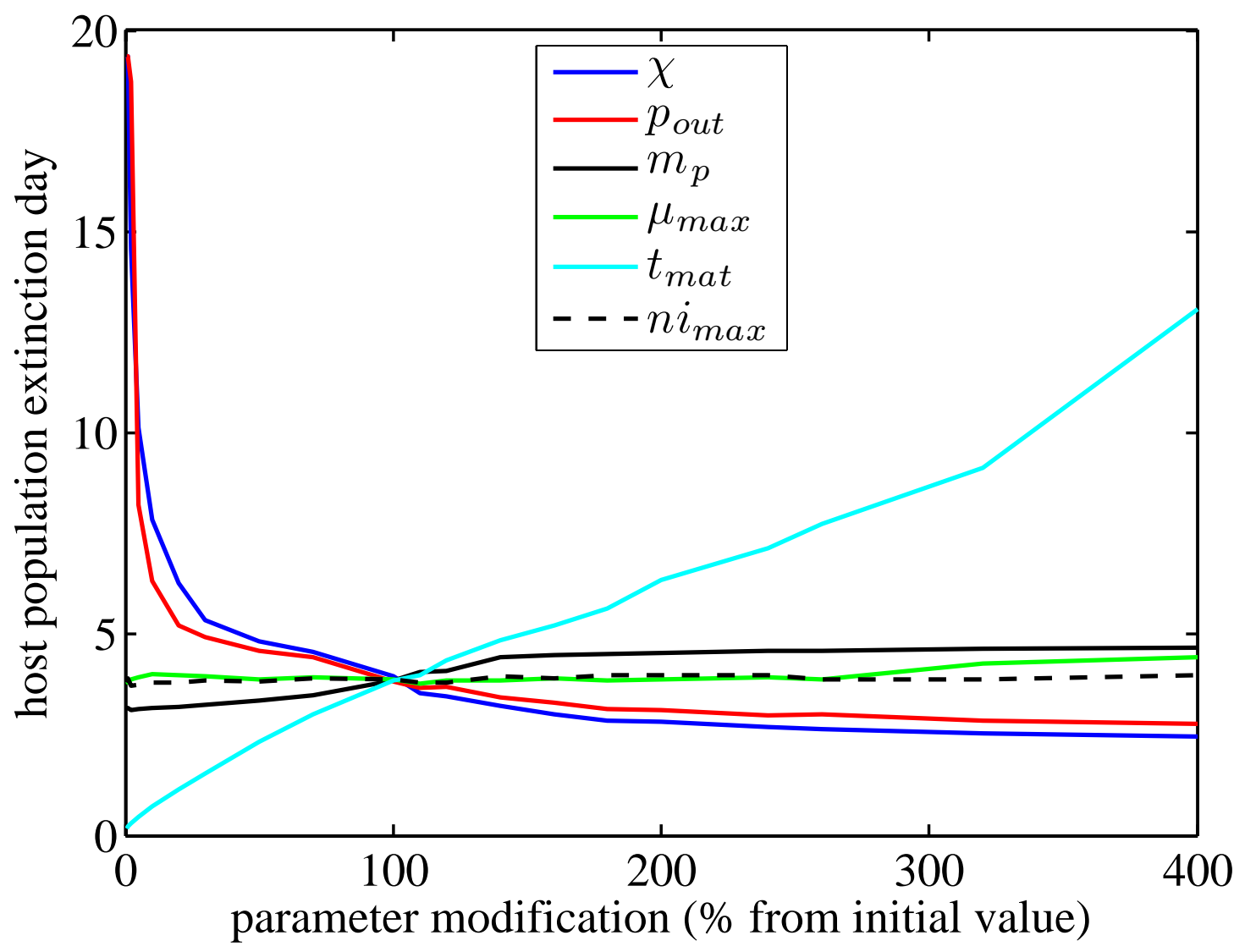

Fig. 5: Diagram of the sensitivity of the host (G. instriatum) extinction time with a 1:1 parasite-host ratio. Six different parameters were tested: parasite fixation probability $(\chi)$, dinospore production $\left(p_{\text {out }}\right)$, dinospore mortality $\left(m_{p}\right)$, host maximum growth rate $\left(\mu_{\max }\right)$, trophont maturation time $\left(t_{\text {mat }}\right)$ and maximum number of multi infections $\left(n i_{\text {max }}\right)$. 
a
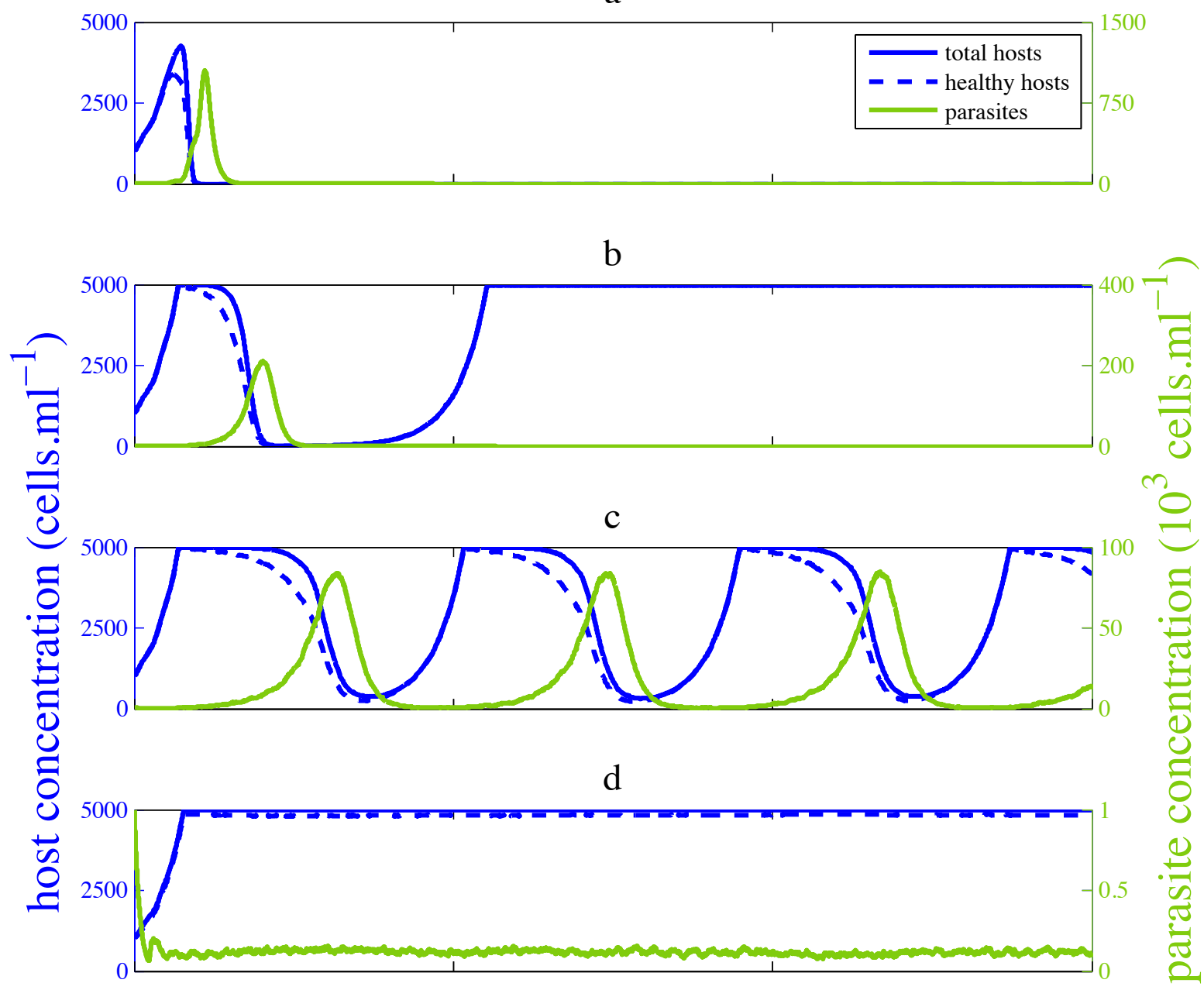

$\mathrm{e}$

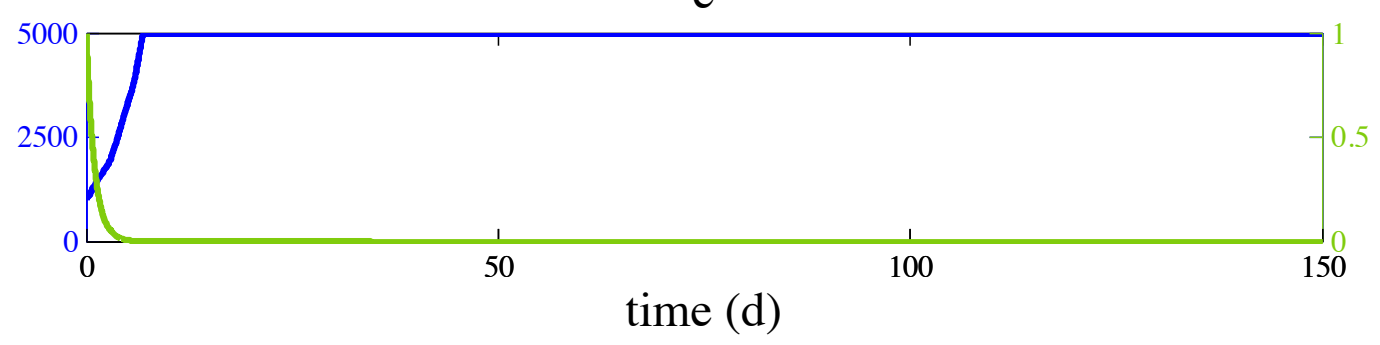

Fig. 6: Illustration of the different dynamics simulated by modifying $\chi$ and $p_{\text {out }}$. Host (blue and blue dashed lines) and parasite (green lines) dynamics for a 150-day simulation with the species G. instriatum. The different dynamics presented are: host extinction (a), parasite extinction post-infection (b), oscillation (c), stable coexistence (d) and parasite extinction (e). 

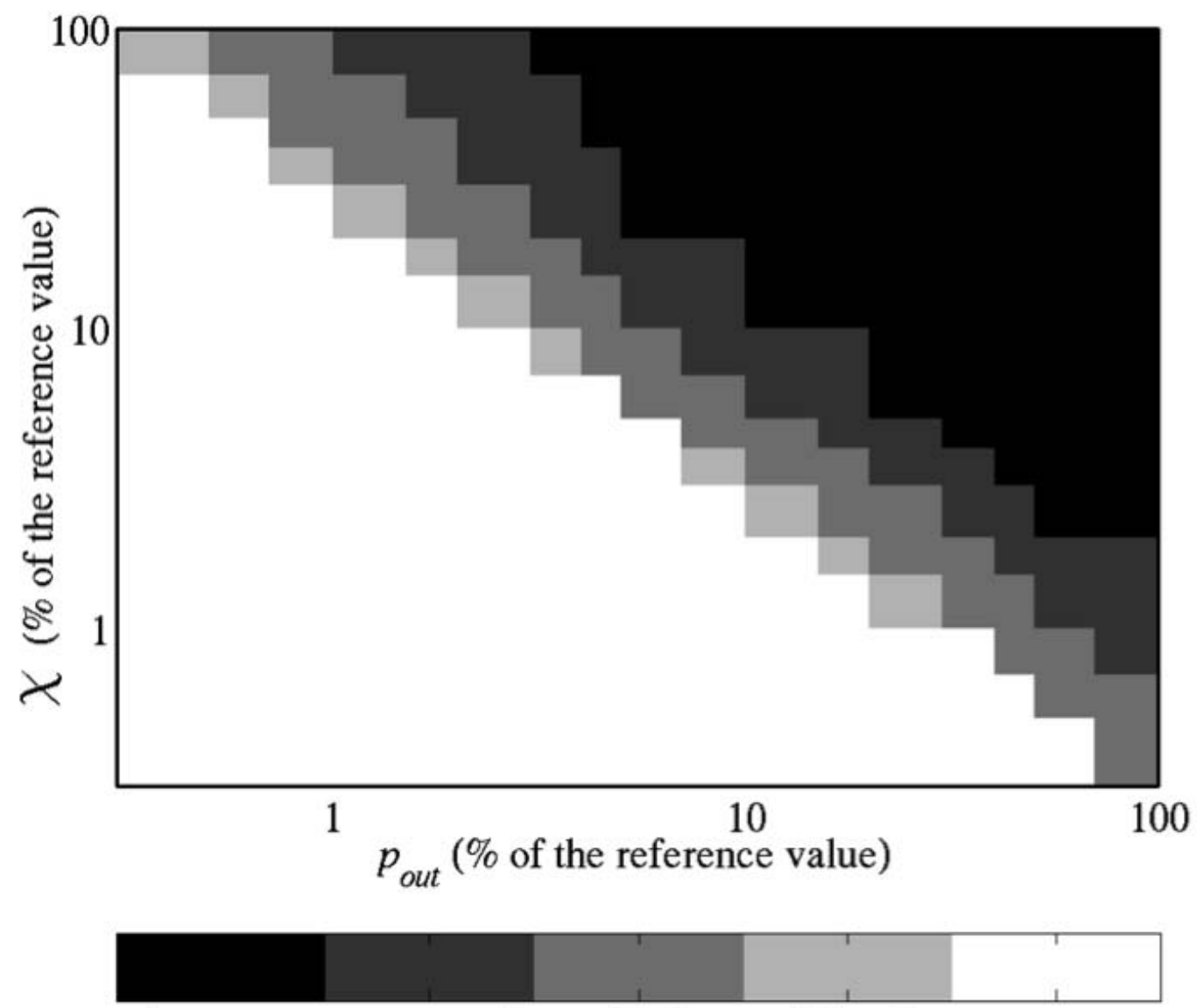

host ext. par. ext. pi. oscillation stabilisation par. ext.

Fig. 7: Diagram of the modifications in dynamics associated with modifications in $\chi$ and $p_{\text {out }}$ for 150-day simulations. The most frequently obtained dynamics from among ten simulations with the species $G$. instriatum and a parasite-host ratio of 1:1 are presented. Five different dynamics were simulated: host extinction (host ext.), parasite extinction post-infection (par. ext. pi.), oscillation, stabilization and parasite extinction (par. ext.). 


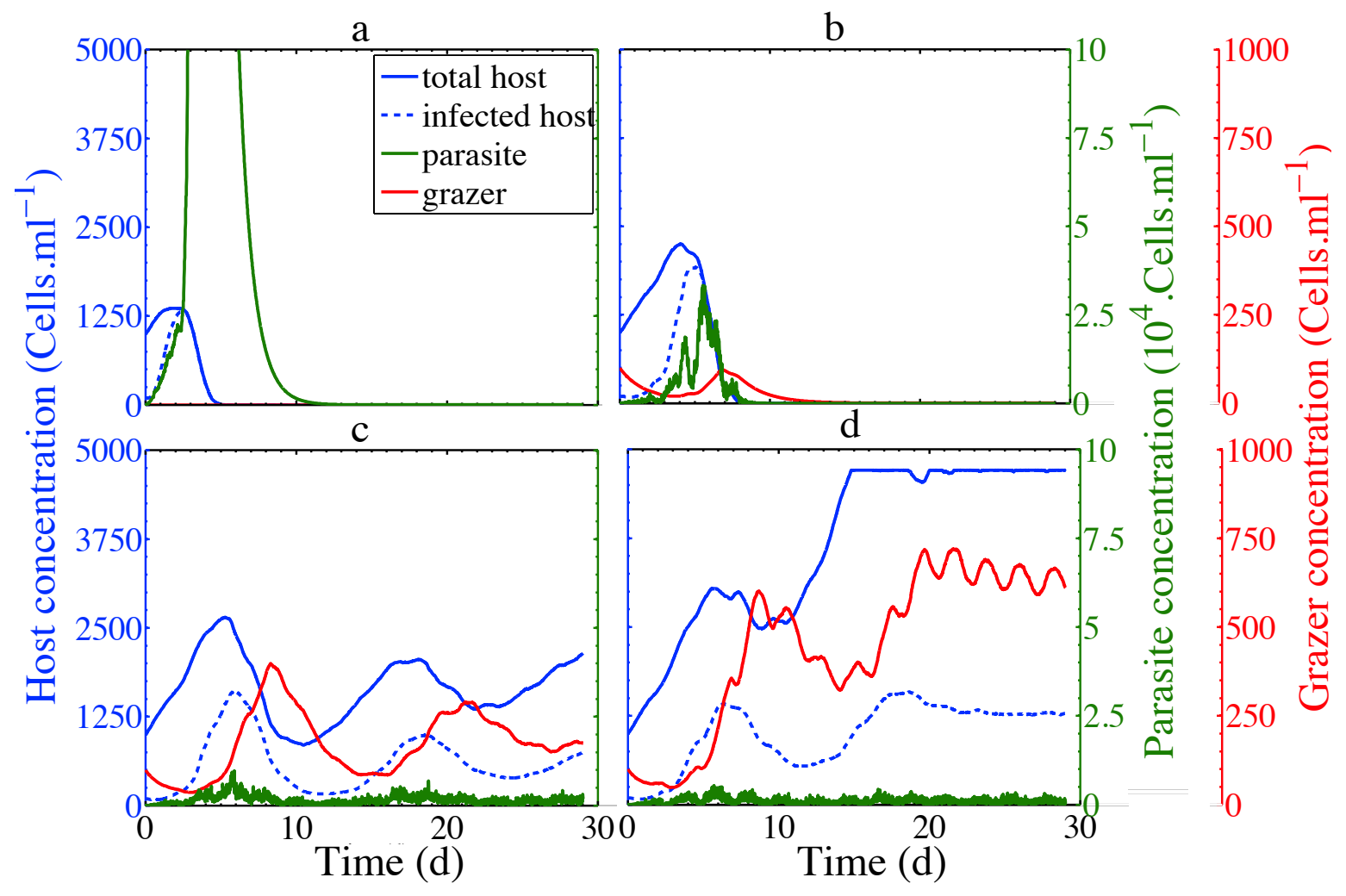

Fig. 8: 30-day simulations of G. instriatum (blue lines and blue dashed lines) infected with Amoebophrya spp. (green) and Strobilidium sp. (red) grazing on the parasite. Simulation without a grazer (a) and with a grazer (b to d). Grazer growth rates $\left(k_{g}\right)$ assume values of $6.26 \times 10^{-5}(\mathrm{~b}), 3.13 \times 10^{-4}$ (c) and $6.26 \times 10^{-4} \mathrm{ml} \cdot$ parasites $^{-1}(\mathrm{~d})$. 


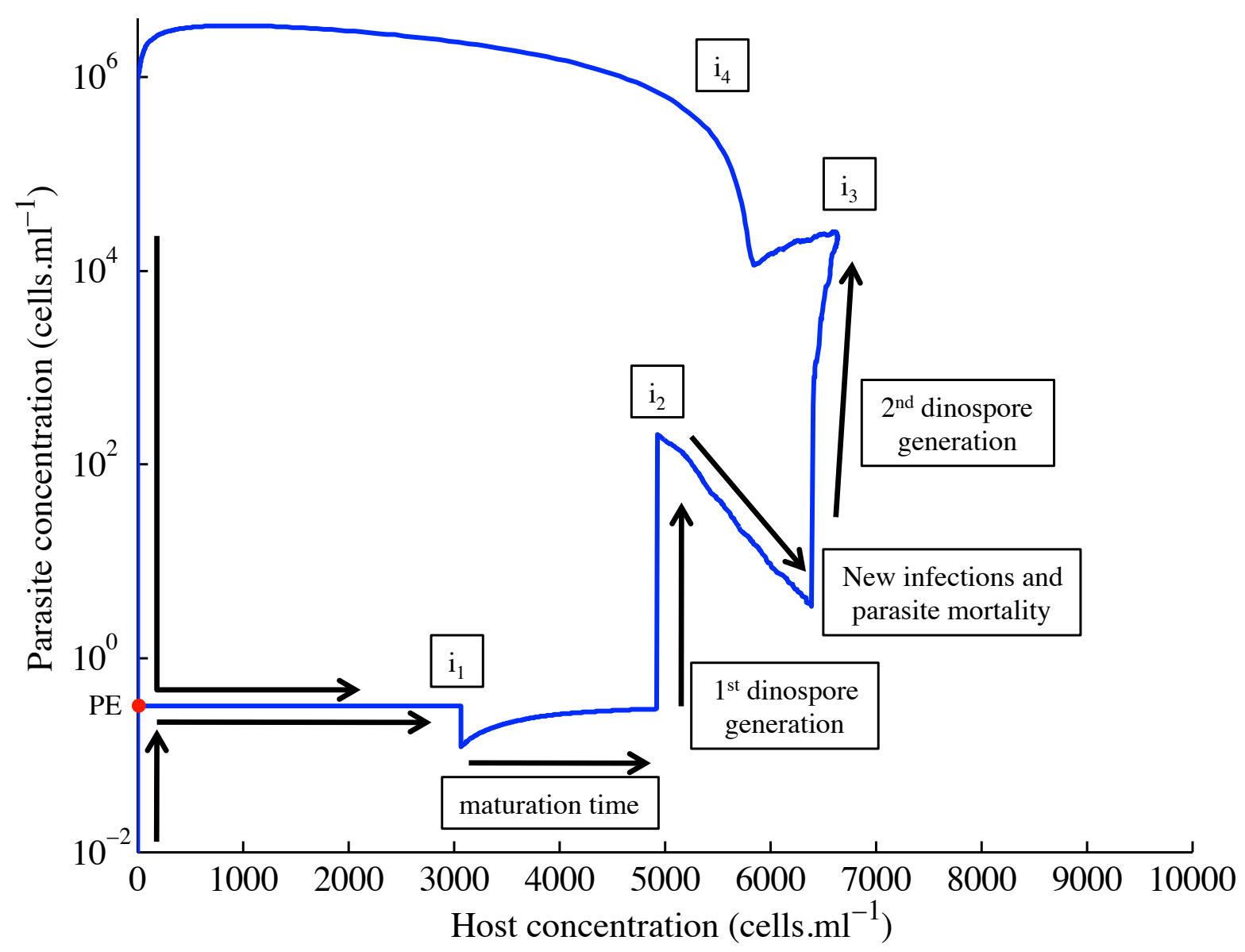

Fig. 9: Phase diagram of the host-parasite dynamics with one oscillation caused by excystment during a 90-day simulation. $\mathrm{PE}$ is the parasite concentration when an equilibrium between production by excystment and mortality is attained. $i_{n}$ corresponds to the $n^{\text {th }}$ wave of infection. 